\title{
Doppler search for exoplanet candidates and binary stars in a CoRoT field using a multi-fiber spectrograph ${ }^{\star}$
}

\section{Global analysis and first results}

\author{
B. Loeillet ${ }^{1}$, F. Bouchy ${ }^{1,2}$, M. Deleuil ${ }^{1}$, F. Royer $^{3}$, J. C. Bouret ${ }^{1}$, C. Moutou ${ }^{1}$, P. Barge ${ }^{1}$, P. de Laverny ${ }^{4}$, F. Pont ${ }^{5}$, \\ A. Recio-Blanco ${ }^{4}$, and N. C. Santos ${ }^{5,6}$ \\ ${ }^{1}$ Laboratoire d'Astrophysique de Marseille, BP 8, 13376 Marseille Cedex 12, Université de Provence, CNRS (UMR 6110) and \\ CNES, France \\ e-mail: benoit.loeillet@oamp. fr \\ 2 Institut d'Astrophysique de Paris, CNRS, Université Pierre et Marie Curie, 98bis Bd Arago, 75014 Paris, France \\ 3 GEPI/CNRS UMR 8111, Observatoire de Paris, 5 place Jules Janssen, 92195 Meudon Cedex, France \\ ${ }^{4}$ Observatoire de la Côte d'Azur, Dpt. Cassiopée, CNRS-UMR 6202, BP 4229, 06304 Nice Cedex 04, France \\ 5 Observatoire de Genève, Université de Genève, 51 Ch. des Maillettes, 1290 Sauverny, Switzerland \\ ${ }^{6}$ Centro de Astrofísica, Universidade do Porto, Rua das Estrelas, 4150-762 Porto, Portugal
}

Received 26 January 2007 / Accepted 26 November 2007

\section{ABSTRACT}

\begin{abstract}
Context. The discovery of the short-period giant exoplanet population, the so-called hot Jupiter population, and their link to brown dwarfs and low-mass stars challenges the conventional view of planet formation and evolution.

Aims. We took advantage of the multi-fiber facilities GIRAFFE and UVES/FLAMES (VLT) to perform the first large radial velocity survey using a multi-fiber spectrograph to detect planetary, brown-dwarf candidates and binary stars.

Methods. We observed 816 stars during 5 consecutive half-nights. These stars were selected within one of the exoplanet fields of the space mission CoRoT.

Results. We computed the radial velocities of these stars and showed that a systematic error floor of $30 \mathrm{~m} \mathrm{~s}^{-1}$ was reached over 5 consecutive nights with the GIRAFFE instrument. Over the whole sample the Doppler measurements allowed us to identify a sample of 50 binaries, 9 active or blended binary stars, 5 unsolved cases, 14 exoplanets and brown-dwarf candidates. Further higher precision Doppler measurements are now necessary to confirm and better characterize these candidates.

Conclusions. This study demonstrates the efficiency of a multi-fiber approach for large radial-velocity surveys in search for exoplanets as well as the follow-up of transiting exoplanet candidates. The spectroscopic characterization of the large stellar population is an interesting by-product of such missions as the CoRoT space mission.
\end{abstract}

Key words. techniques: radial velocities - instrumentation: spectrographs - stars: binaries: spectroscopic stars: low-mass, brown dwarfs - stars: planetary systems

\section{Introduction}

The discovery of an ever increasing sample of extra-solar planets (at present more than 250 objects have been detected) shows remarkable objects very close to their parent star. This exoplanet population, called the hot-Jupiter population, is composed of giant planets that revolve at a short orbital distance around a central star. They are orbiting at less than 0.1 astronomical units (AU) with an orbital period ranging from 1.2 to 10 days. Today, more than 40 members of this population have been detected. This existance of this population suggests new mechanisms of planet formation and evolution, not envisioned in the study of our Solar System, such as the migration of the planets in the proto-planetary disk or gravitational interactions (Goldreich \& Tremaine 1980; Lin et al. 1996).

Mechanisms of planetary formation can be investigated via the mass function by exploring the gap which separates the highmass "planetary" companions (considered here to be more than

\footnotetext{
* Based on observations collected with the GIRAFFE and UVES/FLAMES spectrographs at the VLT/UT2 Kueyen telescope (Paranal observatory, ESO, Chile: program 074.C-0633A).
}

$M \sin i=3 M_{\text {Jup }}$ ) from their low-mass "stellar" counterparts. The "super-planet" or brown dwarf HD 162020b (Udry et al. 2002), the super-massive planet HD 147506b (Bakos et al. 2007) and the very low-mass stars OGLE-TR-122,123 (Pont et al. 2005, 2006) are at the tails of these 2 populations and illustrate this gap. These suggest two different processes of formation and evolution of planetary systems. High-mass exoplanets and lowmass stellar companions with short periods are quite rare. We can only list 6 examples of high-mass exoplanets with an orbital period of less than 20 days: Gl86b (Queloz et al. 2000), Tau Boo b (Butler et al. 1997), HIP 14810b (Wright et al. 2006), HD 195019b (Fischer et al. 1999), HD 162020b (Udry et al. 2002) and HD 147506b (Bakos et al. 2007). All of them have a minimum mass between 3.5 and $14 M_{\mathrm{Jup}}$.

Ground-based photometric transit surveys such as OGLE (Udalski et al. 2002), SuperWASP (Pollacco et al. 2006), TrES (Alonso et al. 2007), HAT (Bakos et al. 2006), have sufficient photometric precision to detect transiting hot Jupiters and eclipsing low-mass stars. New space missions, designed to search for transiting exoplanets, will probe the planetary population at smaller radii than ever before thanks to an improved photometric 
precision, a longer temporal coverage with a better duty cycle, and without the observational perturbations induced by the Earth's atmosphere. In parallel, the next step for radial-velocity (RV) programs consists of simultaneously observing a large sample of stars with a reduced on-telescope time. This could be foreseen by using multi-fiber technology combined with accurate spectrographs.

In a first attempt, Bouchy et al. $(2004,2005)$ have demonstrated the ability of the UVES/FLAMES spectrograph to obtain precise Doppler measurements. However, only 7 stars could be monitored at the same time with their instrumental configuration. This may be convenient for the Doppler follow-up of planetary candidates but is not sufficient for a large Doppler survey to detect new extra-solar planets with limited telescope time. With more than 100 fibers distributed over a large field of view of 25 arcmin, the GIRAFFE spectrograph at the VLT (Pasquini et al. 2000), used in the MEDUSA mode may overcome this limitation. However, its ability to accurately measure Doppler shifts has yet to be demonstrated.

In January 2005, we performed a dedicated radial-velocity survey of more than 800 stars with the GIRAFFE and the UVES/FLAMES spectrographs. We chose to observe one of the exoplanet fields of the space mission CoRoT, launched in December 2006 (Baglin 2003). We took advantage of the target characterization of the exoplanet input catalog which provides an approximate spectral classification and astrometry of the stars (Moutou et al. 2007, in prep.). Our scientific goals were to :

- identify massive hot-Jupiter exoplanet and brown dwarf candidates;

- identify binary stars in a CoRoT field;

- explore the ability of the multi-fiber instrument GIRAFFE to accurately measure Doppler shifts for more than one hundred stars simultaneously;

- check and further improve the spectral classification in the CoRoT exoplanet fields by photometric observations.

In this paper, we present the results of our radial velocity analysis carried out on 816 stars. The spectral analysis of the whole sample of spectra will be the subject of a separate paper. The sample of stars is presented in Sect. 2. The data reduction method and the subsequent RV measurements are detailed in Sect. 3. The $\mathrm{RV}$ performance is discussed in Sect. 4 and the first results of the radial velocity analysis are presented in Sect. 5 .

\section{Observations}

\subsection{FLAMES facilities}

In January 2005 we obtained 5 half-nights in visitor mode with the FLAMES facilities (program 074.C-0633A) attached to the $8.2 \mathrm{~m}$ Kueyen telescope (UT2) based at the ESO-VLT. FLAMES is a multi-fiber link which makes it possible to feed up to 130 targets into the GIRAFFE echelle spectrograph which covers a 25 arcmin diameter field-of-view $\left(0.136^{\circ 2}\right)$. In addition, a simultaneous Thorium-Argon calibration is available by using the MEDUSA mode which dedicates 5 fibers to monitor a Th-Ar lamp. The fiber link allows a stable illumination at the entrance of the spectrograph and the simultaneous calibration is used to track instrumental drift.

We chose to observe in the HR9B spectral domain, centered at $525.8 \mathrm{~nm}$. This setup covers $200 \AA$ and a CCD pixel corresponds to $0.05 \AA$. This instrumental configuration offers the highest resolution $(R=25800)$ and the best radial velocity accuracy (see Royer et al. 2002). It indeed provides the best compromise in term of signal-to-noise and the number of spectral lines necessary for accurate Doppler measurements.

We combined the GIRAFFE spectrograph with $U V E S / F L A M E S$ which allow us to simultaneously observe 7 additional stars at a much higher resolution plus one fiber dedicated to the simultaneous Thorium calibration. Observations were made with the red arm of the spectrograph at a central wavelength of $580 \mathrm{~nm}$. This setup covers $2000 \AA$ with a resolution of 47000 and a CCD pixel corresponds to $0.015 \AA$.

\subsection{Target selection and observational strategy}

We chose to observe stars selected in one of the exoplanet CoRoT fields located in a region close to the direction of the Galactic anti-center. This field has a homogeneous star density of about 3600 stars per square degree with magnitudes brighter than $r<15$. It will be one of the first fields to be observed by the space mission during the first year of operation.

We chose 6 different FLAMES fields, with no overlap between each field, and distributed over an area that covers about $40 \%$ of one of the two CoRoT exoplanet CCDs. This results in a total of 774 stars observed with the GIRAFFE spectrograph plus 42 additional ones observed at a higher resolution with UVES/FLAMES.

When selecting our targets in these FLAMES fields, we took advantage of the CoRoT exoplanet entry catalogue EXODAT (Deleuil 2006). Built from dedicated ground-based observations and existing catalogues to prepare the exoplanet program, this catalogue not only provides the astrometry for all stars within the potential exoplanet fields of the mission, but also gives an estimate of the spectral types and luminosity classes of all the stars in the range 11 to 16 in $r$-mag. Thanks to this information, the target selection was conducted according to 3 different levels of priority, depending on our scientific goals and on the instrumental constraints: angular separation between stars for the positioning of the fibers, field of view of the instrument, etc. Our 3 levels of priorities were defined as: 1) bright solar-type stars (F, G and K spectral type stars) and a $V$-mag less than $15 ; 2$ ) F0 to $\mathrm{K} 5$ giant stars and stars with a $V$-mag greater than 15 ; 3 ) $\mathrm{O}$, $\mathrm{B}$ and A-type stars, and giant stars with spectral type ranging from $\mathrm{K} 5$ to $\mathrm{M}$. The $U V E S / F L A M E S$ fibers were allocated to targets of the first priority only.

Figure 1 shows the number of stars as a function of $V$ magnitude for the two spectrographs. Figure 2 shows the number of stars as a function of spectral type for the two main luminosity classes, again for the two spectrographs. The majority of the fibers have thus been allocated to F, G, K-type dwarfs, which are most favorable for precise radial velocity measurements. They represent about $60 \%$ of our targets. The remaining targets are mainly giants (30\%) and A-type dwarfs (10\%).

The observational strategy consists of one measurement per night of each of the 6 fields during the 5 consecutive half-nights with a typical exposure time of $30 \mathrm{~min}$. One of our fields was observed only 3 times, due to a lack of observational time during 2 of the 5 nights. The journal of the observations is presented in Table 1 . We have obtained 5 different spectra for 680 stars and 3 spectra for 136 stars, which results in a total of 3808 spectra.

We took care to keep the same configuration each night. Each star was allocated to the same fiber on the instrument on each of the 5 consecutive nights in order to minimize errors, which could be due to different chromatic behavior of the fibers and/or different pixel responses on the CCD. 
Table 1. Journal of the observations with the coordinates of the field centers and the exposure time used.

\begin{tabular}{ccccccc}
\hline \hline & Field 0 & Field 1 & Field 2 & Field 3 & Field 4 & Field 5 \\
\hline$\alpha$ center & $6: 42: 26$ & $6: 42: 26$ & $6: 42: 26$ & $6: 44: 06$ & $6: 44: 06$ & $6: 44: 06$ \\
$\delta$ center & $-1: 23: 51$ & $-0: 52: 30$ & $-0: 27: 30$ & $-1: 17: 30$ & $-0: 52: 30$ & $-0: 27: 30$ \\
MJD - 53 393 / & 1.0225 & 1.1865 & 1.0526 & 1.0876 & 1.1203 & 1.1538 \\
Exposure time (min) & 30 & 30 & 35 & 35 & 35 & 35 \\
& 2.0228 & - & 2.0651 & 2.1368 & 2.1741 & 2.2041 \\
& 49 & - & 30 & 42.3 & 30 & 30 \\
& 3.0249 & 3.1879 & 3.0583 & 3.0912 & 3.1234 & 3.1559 \\
& 35 & 25 & 35 & 35 & 35 & 35 \\
& 4.0214 & - & 4.0544 & 4.0877 & 4.1197 & 4.1524 \\
& 35 & - & 35 & 35 & 35 & 35 \\
& 5.0194 & 5.1666 & 5.0481 & 5.0774 & 5.1062 & 5.1374 \\
& 30 & 25 & 30 & 30 & 30 & 30 \\
\hline
\end{tabular}

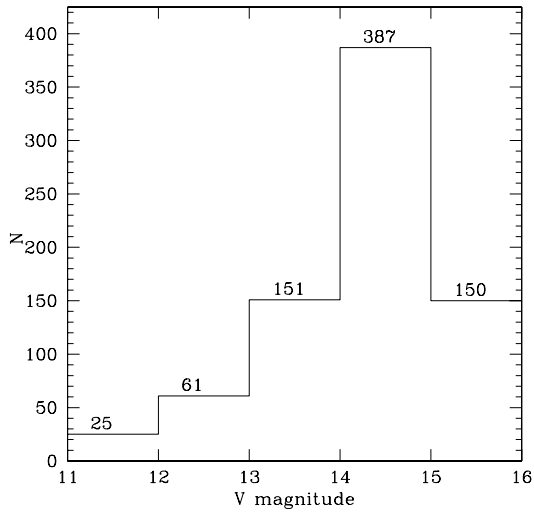

(a)

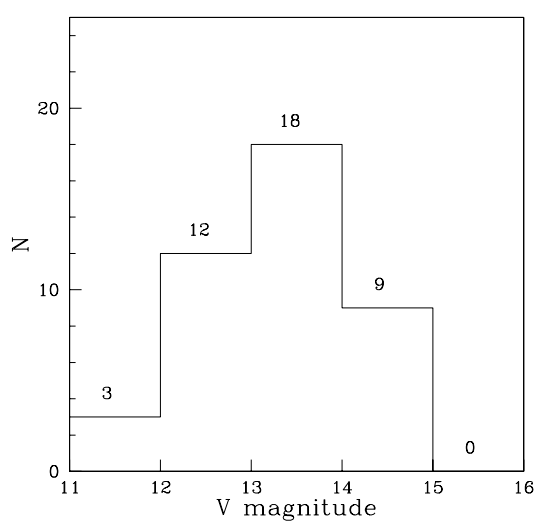

(b)

Fig. 1. Magnitude distribution of the observed stars with the GIRAFFE (a)) and the UVES/FLAMES (b)) spectrographs.

\section{Data reduction}

In the next sections we describe the essentials of the extraction and reduction process adopted for the GIRAFFE data. The one used for the UVES/FLAMES data is detailed in Bouchy et al. (2004).

\subsection{Extraction and wavelength calibration}

The frames have been reduced using the GIRAFFE BaseLine Data Reduction Software (girbldrs v1.12, see Blecha et al. 2000;

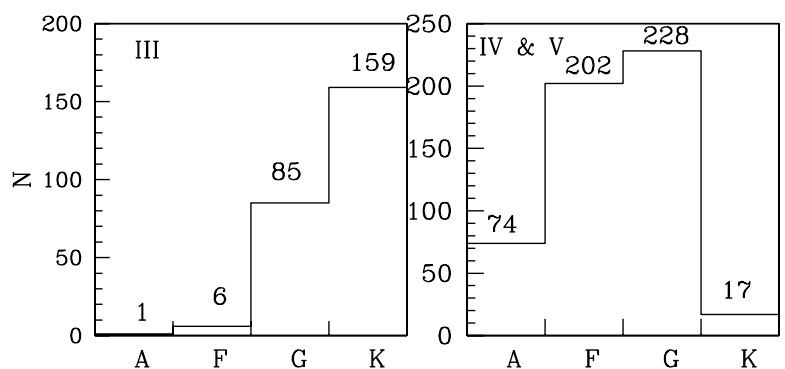

(a)
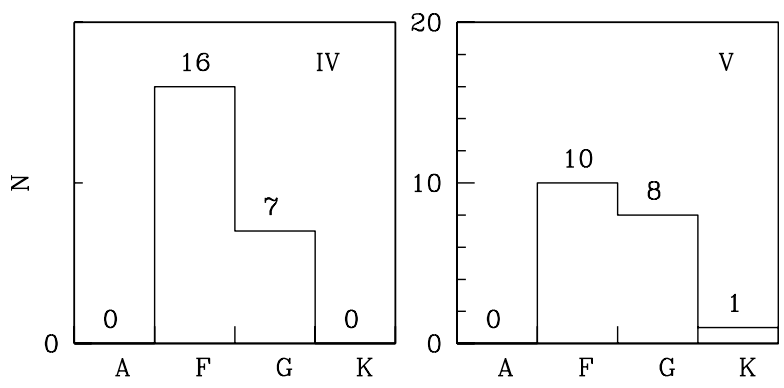

(b)

Fig. 2. Distribution of the sample of observed stars with the GIRAFFE a) and the UVES/FLAMES; b) spectrograph over the spectral type for dwarfs and giants. The sample is completed by a O-type dwarf star and a M-type giant star.

Royer et al. 2002). The data were optimally extracted to 1D spectra following the original Horne's method (Horne 1986). Analyzing the Th-Ar calibration spectra, we found an instability of the zero-point of the absolute wavelength calibration along the calibrations made during the 5 nights. To set the zero-point, we chose to use the Th-Ar calibration spectra registered during the first day of our observational campaign. The wavelength solution was thus computed once, using this first Th-Ar exposure and applied to all the scientific frames. This allowed for a better estimation of the instrumental drift between observation nights and observational exposures thanks to the simultaneous Th-Ar spectra (see Sect. 3.4 for more details). We thus used the simultaneous Th-Ar spectra for each stellar observation to correct the drift from the first Th-Ar calibration exposure. 


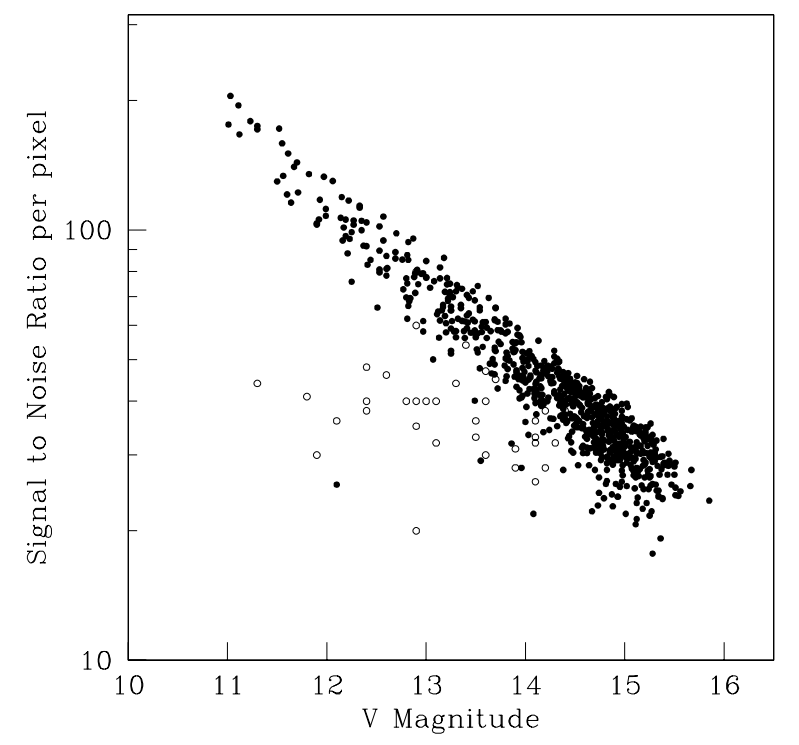

Fig. 3. Signal-to-noise ratio per pixel of all the spectra measured on the last observation night as a function of stellar magnitude. The GIRAFFE data is shown by filled circles ( 1 pixel $=0.05 \AA$ ) and the UVES/FLAMES data is shown by open circles ( 1 pixel $=0.015 \AA$ ). The signal-to-noise scale is a logarithmic scale and we note that both parameters are linked by a clear logarithmic relation.

\subsection{Radial velocity measurements}

Radial velocities were obtained by a weighted cross-correlation (Baranne et al. 1996) of each spectrum with a numerical mask, constructed from the Solar spectrum atlas (Baranne et al. 1996; Pepe et al. 2002). The resulting Cross-Correlation Function (CCF) exhibits a Gaussian shape. By fitting it by a Gaussian function, we derived the full width half maximum ( $F W H M$ in $\mathrm{km} \mathrm{s}^{-1}$ ) which gave an estimate of the projected rotational velocity of the star, the depth $(\mathrm{C}$ in $\%)$ and the radial velocity ( $\mathrm{RV}$ in $\mathrm{km} \mathrm{s}^{-1}$ ) given by the center of the Gaussian. In our study, we used the same $\mathrm{G} 2$ template mask for the UVES/FLAMES and the GIRAFFE spectra. It is adapted for most solar-type stars. We performed the same analysis with different masks which gives no significant improvements on the RV determination (see Sect. 5). Finally we corrected the calculated RV by the barycentric Earth RV.

\subsection{Photon noise analysis}

Our RV measurements are not photon-noise limited. They depend on the photon noise and an instrumental error floor set by systematic effects. This error floor clearly appears for stars with a short RV variation, as illustrated by Fig. 4a. Small observed RV dispersion (rms) values are indeed set around an average value much larger than $0 \mathrm{~m} \mathrm{~s}^{-1}$. The floor error is set by the asymptotic limit for these stars where the photon noise has very little impact. We estimated this error floor to be equal to $30 \mathrm{~m} \mathrm{~s}^{-1}$. The same level of systematic effects was found for UVES/FLAMES (see Bouchy et al. 2005). Following the analysis carried out by Bouchy et al. (2005), we computed the photon noise uncertainties through the following relation:

$\sigma_{R V}=\sigma_{0} * \frac{\sqrt{F W H M}}{S N R * C}$

where $S N R, F W H M$ and $C$ are respectively the signal-to-noise ratio per pixel, the full width half maximum and the depth of the CCF peak. The constant $\sigma_{0}$ was determined empirically. To evaluate the impact of the photon noise and the value of $\sigma_{0}$ for the GIRAFFE spectrograph, one must move into the regime of large RV rms, where the floor error only enters weakly. The $\sigma_{0}$ values were adjusted so that for the high RV dispersion values the photon noise uncertainty statistically matches the measured $\mathrm{RV}$ rms dispersion. It is equal to 3 for $U V E S / F L A M E S$ and to 10.5 for GIRAFFE. These values are related to the difference in resolution, spectral coverage and the total efficiency of the spectrographs.

\subsection{Simultaneous instrumental drift correction}

For the whole set of spectra, we computed the simultaneous instrumental drift of a single exposure, using the $5 \mathrm{Th}$-Ar fibers. We found that the two extreme Th-Ar fibers, located near the edges of the GIRAFFE CCD, present a significant drift departure, indicating that the wavelength calibration provides less constraint on fibers at the edge on the CCD. We thus used the averaged value of the 3 central Th-Ar fibers of each individual exposure to correct the instrumental drift of the individual exposure with the Th-Ar wavelength calibration. Typically the correction applied is about few hundred $\mathrm{m} \mathrm{s}^{-1}$. We checked that the correction made with the average value of the $5 \mathrm{Th}$-Ar fibers does not drastically change the drift correction in our whole analysis.

\subsection{Background light correction}

Spectra acquired during the first three nights were contaminated by the background light due to the Moon. In particular, the Moon produces an additional peak in the CCF which affects RV measurement when the star's RV is close to the Moon's. We found that about $20 \%$ of our targets are affected in this way. We defined the following procedure to correct for the Moon light in our spectra. For each FLAMES field we selected one spectrum that presents a clear background Moon light and a low SNR stellar spectrum with very shallow spectral lines. We then subtracted this spectrum from all the spectra obtained during the same exposure. We checked that this correction clearly allows us to reduce the RV dispersion for the sample of affected targets. However the correction is not efficient for small RV variations (less than $200 \mathrm{~m} \mathrm{~s}^{-1}$ ). Indeed we noted that the difference between the calculated barycentric correction of the two extreme fibers positioned from the East to the West and distant by about 25 arcmin, reaches $200 \mathrm{~m} \mathrm{~s}^{-1}$ for the same exposure. The observed background light component is then affected in a similar way. The corresponding peak in the CCF is thus shifted in RV. This demonstrates that a single fiber dedicated to sky monitoring is not sufficient. However, we will see in Sect. 5 that this only affects a small fraction of the RV variable stars. One solution to optimize the sky monitoring would be to allocate several fibers in the $\alpha$ direction to limit the difference of the calculated barycentric correction between sky-monitoring fibers and scientific fibers.

\section{Radial velocity performance}

The CCF of 115 stars, representing about $14 \%$ of the whole sample, exhibits no significant correlation peak. Different kind of situations could generate a no-CCF: i) spectra with too small a SNR, ii) stars with a very large $v \sin i$ (typically $>50 \mathrm{~km} \mathrm{~s}^{-1}$ ), iii) early type stars whose stellar features do not match the template well and iv) multiple-line spectroscopic binaries whose 

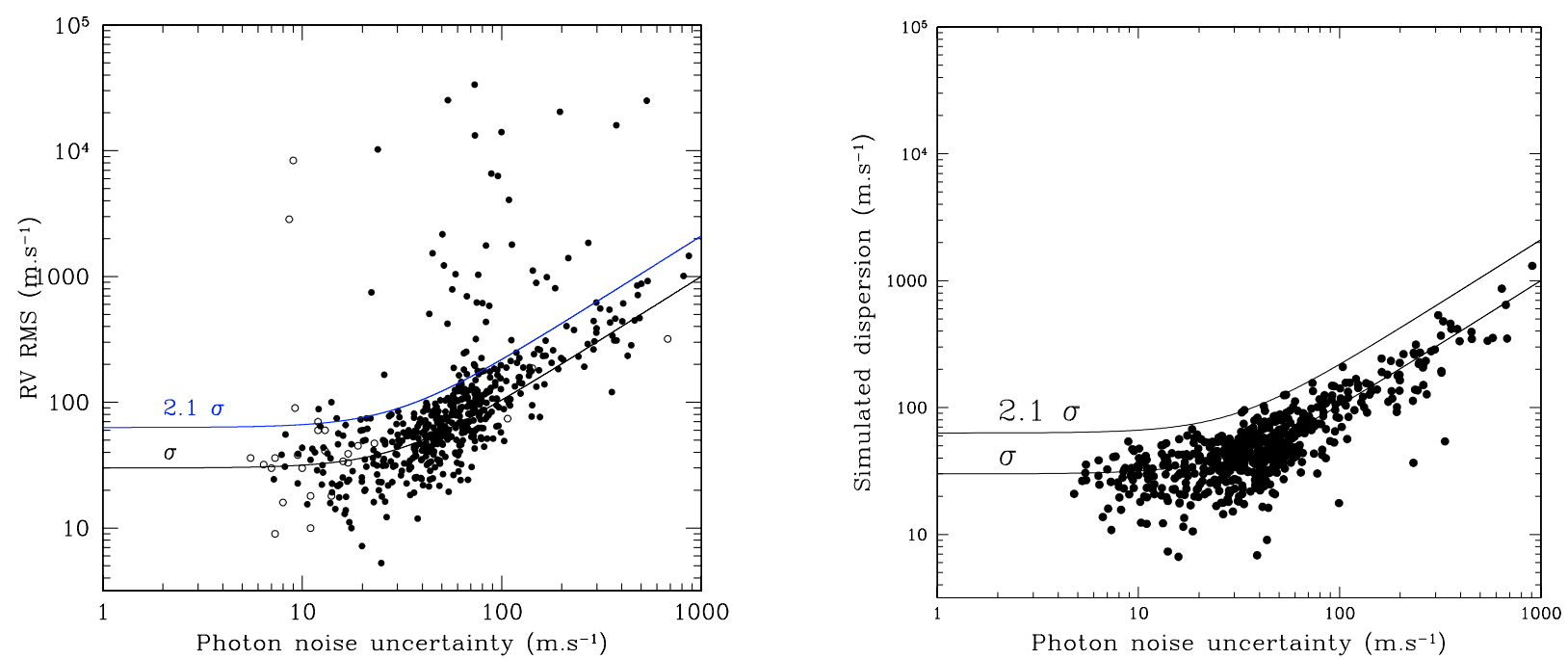

Fig. 4. Distribution of the RV dispersion as a function of photon-noise uncertainty for the GIRAFFE targets in first panel and for simulated dispersions in the second panel. Both scales are logarithmic. The filled circles represent the measurements obtained with the GIRAFFE instrument and the open circles represent the ones obtained with the UVES/FLAMES instrument. The $\sigma$ curve represents the estimated detection limit at $1 \sigma$ of the studied sample of stars. The $2.1 \sigma$ curve shows our threshold to detect RV variable stars. It is derived from simulations presented in the second panel. Stars with identified composite spectra (multiple spectroscopic binaries) have been removed from this figure.

Table 2. Sample of stars observed 3 or 5 times.

\begin{tabular}{ccc|cc}
\hline \hline & \multicolumn{2}{l}{ GIRAFFE targets } & \multicolumn{2}{l}{ UVES/FLAMES targets } \\
& With & No & With & No \\
& CCF & CCF & CCF & CCF \\
\hline 5 meas. & 550 & 95 & 34 & 1 \\
3 meas. & 110 & 19 & 7 & 0 \\
Total & 660 & 114 & 41 & 1 \\
\hline
\end{tabular}

resulting CCF peaks of each component are blended, reducing the contrast of their peak. We checked that among this set of targets with no CCF peak, there is no correlation with the magnitude of the target nor its position within the slit of the instrument. The spectral type of almost all these stars is in the range F8 to O9 and thus they are likely to be rapidly rotating (Cox 2000). We computed the CCF for these specific stars with an F0 template mask which gave no improvement. Our dynamical radial velocity study was thus restricted to the stars yielding a CCF peak. We also narrowed the sample to the stars observed 5 times either with the GIRAFFE or the UVES/FLAMES spectrographs. This results in a total of 584 targets suitable for our radial velocity analysis (Table 2).

We computed the dispersion of the $5 \mathrm{RV}$ measurements and plotted them as a function of the average photon noise uncertainty. Figure 4a displays the RV dispersion (rms) as a function of the estimated photon noise uncertainty for each of these stars. We have excluded stars with composite spectra (multiple spectroscopic binaries). The photon noise uncertainties range from 7 to $700 \mathrm{~m} \mathrm{~s}^{-1}$ and the $\mathrm{RV}$ dispersion ranges from $5 \mathrm{~m} \mathrm{~s}^{-1}$ to $100 \mathrm{~km} \mathrm{~s}^{-1}$.

In order to distinguish the real RV variations of stars from variations generated by statistical effects, we performed a Monte-Carlo simulation. We generated 5 simulated RV for each value of the measured photon noise uncertainty. For that purpose, we used 2 Gaussian distributions with a mean value of zero and a standard deviation equal to the photon noise for the first one and to a systematic error of $30 \mathrm{~m} \mathrm{~s}^{-1}$ for the second one. We then computed the corresponding dispersion of these 5 random values. The results of these simulations are shown in
Fig. 4b. They show that a threshold of $2.1 \sigma$ corresponds to one false detection due to statistical effects over the whole sample.

\section{Radial velocity variation analysis}

Among the set of 701 stars with detected peaks in the CCF and observed 3 or 5 times, we identified 23 spectroscopic binary stars with multiple spectral components in their CCF. Among the set of 584 stars with CCF peak and observed 5 times with the GIRAFFE and UVES/FLAMES spectrographs, our dynamical analysis (see Fig. 4a) allows us to identify 66 targets with a RV dispersion greater than the $2.1 \sigma$ threshold. These targets include spectroscopic binaries of type 1 (SB1), blended binary stars (two merged CCF peaks), active stars and low-mass companion candidates (brown dwarf and exoplanet candidates). The correction of the background light due to the Moon is not totally efficient and some stars are still affected as described in Sect. 3.5. We identified and removed 13 of these 66 targets that are affected by the incomplete Moon light correction. For the remaining 53 stars we looked for an orbital solution by fitting the $5 \mathrm{RV}$ measurements with a sinusoidal curve. We derived the semi-amplitude $K$, the period $P$, the systemic velocity $V_{0}$, and the phase $T_{0}$. Five RV measurements are insufficient to fit an eccentric orbit, therefore we assumed circular orbits. This assumption is justified for orbital periods shorter than 5 days, as the orbit is expected to be circularized by tidal effect (Halbwachs et al. 2005; Marcy et al. 2005). To compute an estimate of the minimum mass of the companion, we derived the stellar masses of these targets using the standard tables of stellar masses (Gray 1988) and the spectral type from the CoRoT exoplanet catalogue.

\subsection{Spectroscopic binary stars}

The so-called spectroscopic binary (SB2) objects show two distinct peaks in their CCF, related to the blended spectra of the two components. Twenty-two SB2 and one clear triple system have been identified. Three SB2 do not present RV variation of both components, probably indicating long-period binary stars. 
Table 3. Binary systems.

\begin{tabular}{|c|c|c|c|c|c|}
\hline $\begin{array}{c}\text { CoRoT } \\
\text { ID }\end{array}$ & $\begin{array}{c}\alpha \\
\text { (h mn s) } \\
(\mathrm{J} 2000)\end{array}$ & $\begin{array}{c}\delta \\
(\mathrm{d} \mathrm{mn} \mathrm{s}) \\
(\mathrm{J} 2000)\end{array}$ & $m_{V}$ & Binary type & $\begin{array}{l}\text { Estimated } \\
\text { period range } \\
\text { (days) }\end{array}$ \\
\hline 102613769 & 64145.38 & -11536.65 & 15.01 & SB1 & $P>50 \mathrm{~d}$ \\
\hline 102614070 & 64145.77 & 03053.32 & 14.98 & SB2 & $P \sim 20 \mathrm{~d}$ \\
\hline 102619385 & 64153.58 & -12336.49 & 14.62 & SB2 & $P \sim 8 \mathrm{~d}$ \\
\hline 102619435 & 64153.64 & -05405.29 & 14.69 & SB1 & Unsolved $P$ ( 3 meas.) \\
\hline 102627314 & 64205.32 & -0 2948.73 & 14.63 & SB1 & $P>100 \mathrm{~d}$ \\
\hline 102627556 & 64205.7 & 02325.19 & 14.67 & SB2 & $P>50 \mathrm{~d}$ \\
\hline 102629764 & 64208.98 & 03130.29 & 14.11 & SB1 & $P<20 \mathrm{~d}$ \\
\hline 102631863 & 64212.06 & -12826.65 & 14.72 & SB1 & $20 \mathrm{~d}<P<50 \mathrm{~d}$ \\
\hline 102634388 & 64216.01 & -04902.82 & 13.55 & SB2 & Unsolved $P$ (3 meas.) \\
\hline 102637099 & 64219.43 & -05300.82 & 14.25 & SB2 & Unsolved $P$ (3 meas.) \\
\hline 102639561 & 64222.68 & -12701.30 & 11.93 & SB2 & $P>100 \mathrm{~d}$ \\
\hline 102646279 & 64231.39 & -04959.05 & 12.41 & SB2 & $P>100 \mathrm{~d}$ \\
\hline 102648472 & 64234.22 & -0 3421.94 & 14.49 & SB1 & Unsolved $P$ (5 meas.) \\
\hline 102648907 & 64234.76 & -10431.22 & 14.53 & SB1 & Unsolved $P$ (3 meas.) \\
\hline 102651332 & 64237.86 & 02430.28 & 15.36 & SB1 & $20 \mathrm{~d}<P<50 \mathrm{~d}$ \\
\hline 102651632 & 64238.21 & 03033.66 & 14.12 & SB2 & $P \sim 15 \mathrm{~d}$ \\
\hline 102652553 & 64239.41 & 03856.29 & 14.89 & SB2 & $P \sim 12 \mathrm{~d}$ \\
\hline 102662997 & 64252.63 & $\begin{array}{lll}-1 & 10 & 10.88\end{array}$ & 12.84 & SB1 & $P \sim 9 \mathrm{~d}$ \\
\hline 102663892 & 64253.77 & -11753.56 & 12.4 & SB1 & $20 \mathrm{~d}<P<50 \mathrm{~d}$ \\
\hline 102668497 & 64259.60 & 02500.91 & 13.30 & SB1 & $P \sim 11 \mathrm{~d}$ \\
\hline 102669579 & 64300.93 & 02623.93 & 15.00 & SB1 & $P \sim 14 \mathrm{~d}$ \\
\hline 102672236 & 64304.28 & -12028.28 & 15.07 & SB2 & $P \sim 12 \mathrm{~d}$ \\
\hline 102673596 & 64306.00 & -11608.00 & 13.69 & SB2 & $P>100 \mathrm{~d}$ \\
\hline 102677302 & 64310.53 & -11200.90 & 13.04 & SB1 & $P \sim 11 \mathrm{~d}$ \\
\hline 102683896 & 64318.80 & -11641.74 & 14.61 & SB2 & $P>50 \mathrm{~d}$ \\
\hline 102686545 & 64322.12 & -11234.70 & 13.96 & SB1 & $P>50 \mathrm{~d}$ \\
\hline 102692263 & 64329.23 & 05633.72 & 14.97 & SB1 & $P \sim 8 \mathrm{~d}$ \\
\hline 102694802 & 64332.34 & -1 1920.35 & 14.47 & SB1 & $20 \mathrm{~d}<P<50 \mathrm{~d}$ \\
\hline 102708308 & 64348.88 & 05908.09 & 15.15 & SB1 & $P \sim 18 \mathrm{~d}$ \\
\hline 102708916 & 64349.76 & 04751.25 & 13.97 & SB2 & $P \sim 5 \mathrm{~d}$ \\
\hline 102709159 & 64350.10 & 04131.02 & 14.00 & SB1 & $P \sim 17 \mathrm{~d}$ \\
\hline 102709968 & 64351.21 & 03454.01 & 14.32 & SB2 & $P \sim 12 \mathrm{~d}$ \\
\hline 102712875 & 64355.22 & 05109.00 & 14.38 & SB2 & $P \sim 16 \mathrm{~d}$ \\
\hline 102713079 & 64355.51 & 04626.69 & 14.73 & SB1 & $20 \mathrm{~d}<P<50 \mathrm{~d}$ \\
\hline 102715243 & 64358.47 & -10051.62 & 13.1 & SB2 & $P>100 \mathrm{~d}$ \\
\hline 102715978 & 64359.54 & -12934.30 & 13.13 & SB2 & $P \sim 4 \mathrm{~d}$ \\
\hline 102716305 & 64359.97 & 04410.32 & 14.56 & SB1 & $P \sim 18 \mathrm{~d}$ \\
\hline 102718650 & 64403.33 & -12124.66 & 13.7 & SB2 & $P \sim 16 \mathrm{~d}$ \\
\hline 102718810 & 64403.56 & 05742.80 & 13.32 & SB1 & $20 \mathrm{~d}<P<50 \mathrm{~d}$ \\
\hline 102720035 & 64405.35 & -11526.89 & 13.57 & SB1 & $P \sim 20 \mathrm{~d}$ \\
\hline 102725454 & 64413.08 & -11736.53 & 13.35 & SB2 & $P>100 \mathrm{~d}$ \\
\hline 102726103 & 64414.30 & -11344.44 & 14.54 & SB2 & $P \sim 4 \mathrm{~d}$ \\
\hline 102732890 & 64423.49 & -11131.70 & 13.79 & SB1 & $P>50 \mathrm{~d}$ \\
\hline 102734591 & 64425.75 & -11729.40 & 14.39 & SB1 & $P \sim 8 \mathrm{~d}$ \\
\hline 102737852 & 64430.25 & 04154.13 & 15.11 & SB1 & $P>50 \mathrm{~d}$ \\
\hline 102738614 & 64431.29 & $\begin{array}{lll}-1 & 1020.86\end{array}$ & 14.45 & SB2 & $P \sim 7 \mathrm{~d}$ \\
\hline 102740955 & 64434.42 & -1199.12 & 13.27 & SB2 & $P \sim 8 \mathrm{~d}$ \\
\hline 102747222 & 64442.72 & 04522.61 & 12.89 & SB1 & $20 \mathrm{~d}<P<50 \mathrm{~d}$ \\
\hline 102748346 & 64444.27 & -12054.64 & 15.07 & SB1 & $P \sim 15 \mathrm{~d}$ \\
\hline 102748356 & 64444.28 & -11808.24 & 12.9 & SB3 & $P \sim 5 \mathrm{~d}$ \\
\hline
\end{tabular}

We also identified 27 SB1, defined as targets with an estimated companion minimum mass that is greater than the canonical sub-stellar limit of $70 M_{\text {Jup }}$ (Chabrier et al. 2000). Two of those identified in the field were observed only 3 times. They present an RV variation greater than $4 \mathrm{~km} \mathrm{~s}^{-1}$. Table 3 presents the characteristics of the central star and a rough estimation of the orbital period of the stellar companion. For periods greater than 20 days, we only give an estimated period range.

A complete analysis of this sample of binary systems will be completed thanks to complementary photometric observations and RV measurements. Indeed the BEST (Rauer et al. 2004) instrument has already observed a large part of the CoRoT fields and will probably bring additional constraints on these systems. In parallel the space mission CoRoT (Baglin 2003) will observe this field by the end of 2007 .

\subsection{Targets with small $R V$ variations}

Besides the SB1 stars, a subset of 28 stars exhibit an RV dispersion above the $2.1 \sigma$ detection threshold, which corresponds to $63 \mathrm{~m} \mathrm{~s}^{-1}$ for small photon-noise uncertainty. According to the orbital solutions that we found, sub-stellar companions may induce these RV variations. We checked the origin of these RV variations by performing a bisector analysis of the CCF profiles. 
Table 4. Characteristics of identified targets with a line-bisector effect. The proposed interpretation is based on CCF mask analysis.

\begin{tabular}{ccccc}
\hline \hline $\begin{array}{c}\text { ID } \\
\text { COROT }\end{array}$ & $\begin{array}{c}\alpha \\
(\mathrm{h} \mathrm{mn} \mathrm{s}) \\
(\mathrm{J} 2000)\end{array}$ & $\begin{array}{c}\delta \\
(\mathrm{d} \mathrm{mn} \mathrm{s}) \\
(\mathrm{J} 2000)\end{array}$ & $m_{V}$ & Proposed interpretation \\
\hline 102619034 & $641: 53.06$ & -02005.35 & 14.95 & Activity \\
102631629 & 64211.71 & 02953.27 & 13.87 & Blended Binary \\
102631928 & 64212.17 & 02838.50 & 15.39 & Activity \\
102700329 & 64339.10 & -01958.87 & 12.81 & Activity \\
102700855 & 64339.69 & 03105.84 & 14.58 & Blended Binary \\
102717173 & 64401.22 & 02756.92 & 14.34 & Blended Binary \\
102723949 & 64410.95 & -11113.24 & 13.96 & Activity \\
102724171 & 64411.26 & -11009.88 & 13.43 & Blended Binary \\
102743523 & 64437.80 & -03417.98 & 11.50 & Blended Binary \\
\hline
\end{tabular}

This allows us to disentangle RV variations due to true companion from stellar activity.

\subsubsection{Possible blended binary stars and stellar activity signature}

By analyzing the CCF profiles of these targets with a bisector analysis we found that 9 of them presented line-bisector variations. These variations could either be induced by a background blended binary (Queloz et al. 2001) or by stellar photometric activity (Santos et al. 2002). We completed our analysis by exploring the behavior of the RV rms of these stars when crosscorrelated their spectra using 4 different masks. We used masks constructed from M4, K0, K5 and F0 type stars. If the RV rms variation is significant we consider the target to be a blended binary system. On the other hand if no variation is found, the stellar activity origin of the RV variation is more appropriate. We thus identified 5 possible blended binary star systems which present a cross-correlation mask effect and 4 active stars (Table 4).

\subsubsection{Substellar companion candidates}

Fourteen candidates present an orbital solution that matches the exoplanet and brown dwarf mass category and that do not show a line-bisector effect nor cross-correlation mask effects. Figure 5 shows the best orbital solution we obtained for those 14 candidates. Tables 5 and 6 present the parameters of the central stars and the estimated orbital parameters.

A detailed spectral analysis of the stellar spectra of the whole sample is currently under-progress and will be presented in a forthcoming paper. We used on our substellar companion candidate spectra an automatic spectral analysis algorithm (Recio-Blanco et al. 2006), developed for the GAIA/RVS spectroscopic instrument (Wilkinson et al. 2005). This also constitutes the preparation for a planned follow-up program aiming at deriving fundamental parameters of the dwarf population in the CoRoT exoplanet fields. This algorithm, MATISSE (MATrix Inversion for Spectral SynthEsis), derives the stellar parameters and measures the abundances of elements present in the spectra. It employs a grid of synthetic spectra covering the observed spectral domain and computed with the same atmosphere models and line lists (see details in Recio-Blanco et al. 2006). For our RV study, we checked that the spectral classification (Tables 4 and 5) of our subset of candidates derived from the photometric observations on the one hand and from spectral analysis on the other hand are in agreement. We used the spectral type determination made with MATISSE to calculate the minimum mass of our sub-stellar companion candidates.
We checked the likelihood of our 14 candidates by comparing the residuals of the sinusoidal and linear fits to the global uncertainty (photon-noise uncertainty quadratically added to the systematic error). A linear fit would suggest a long-period orbiting object. In most cases, the residuals of the sinusoidal and linear fits are comparable to, or even less than the global uncertainty. Three candidates exhibit a residual much larger than the global uncertainty which could be the signature of an eccentric orbit or stellar activity. The results are presented in Table 6. It is statistically difficult in most cases to disentangle the sinusoidal and linear solutions. They are both compatible with their global uncertainty. However 4 candidates, namely CoRoT ID 102660283, 102666192, 102726906 and 102689702 (in bold type in Table 6), present significant sinusoidal solutions that are statistically better than a linear solution. They appear as our best candidates. One is in the planetary mass range and 3 are in the BD mass range. Taking into account the BD desert (Grether \& Lineweaver 2006), our BD candidates may also be longer-period stellar companions.

\subsubsection{Unsolved orbital solution}

From the sample of 28 stars, 5 targets remain above the detection threshold with small RV variations and unsolved orbital solutions.

Two of them, CoRoT ID 105936100 and 102724641, do not present any satisfactory orbital solutions and their RV variations could be related to stellar activity. The three other targets, namely CoRoT ID102622204, ID102638630 and ID102653533, exhibit large variations of the contrast of their CCF. We checked that these variations are not due to a variation of the SNR nor to fiber-to-fiber contamination. Using a cross-correlation mask analysis, we ruled out a blended binary scenario as the origin of this phenomena.

\section{Discussion}

\subsection{Detection capability of the GIRAFFE instrument}

We performed Monte-Carlo simulations in order to illustrate the capability of the GIRAFFE spectrograph to detect binary stellar systems and exoplanets with 5 consecutive RV measurements. For different stellar companion masses and periods and a solarmass parent star, we computed the RV of 5 consecutive dates, separated by one day, for which the first one was randomly initiated over the period with a random initial phase. We quadratically added a random noise to the systematic error. This noise corresponds to the estimated photon noise uncertainty given a detection threshold thanks to the $\sigma$ curve determined previously for the GIRAFFE instrument. This photon noise uncertainty 
B. Loeillet et al.: Doppler search for exoplanets and binaries using a multi-fiber spectrograph.I.
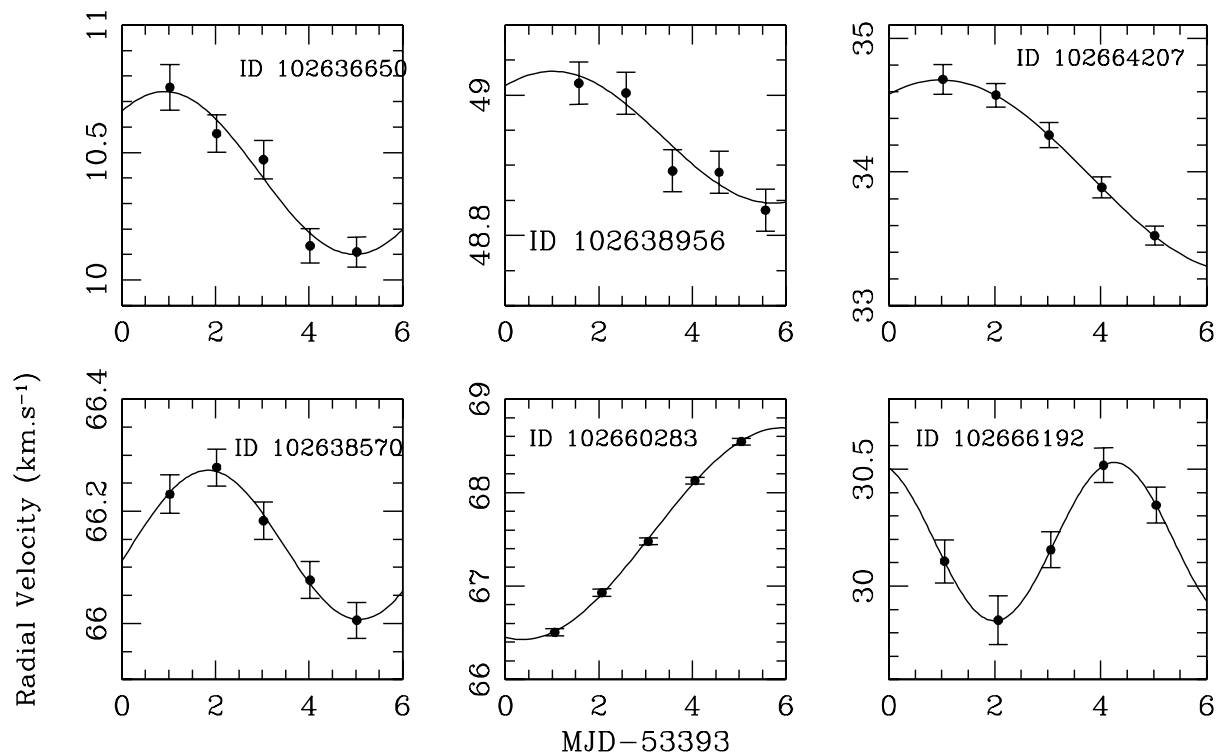

(a)
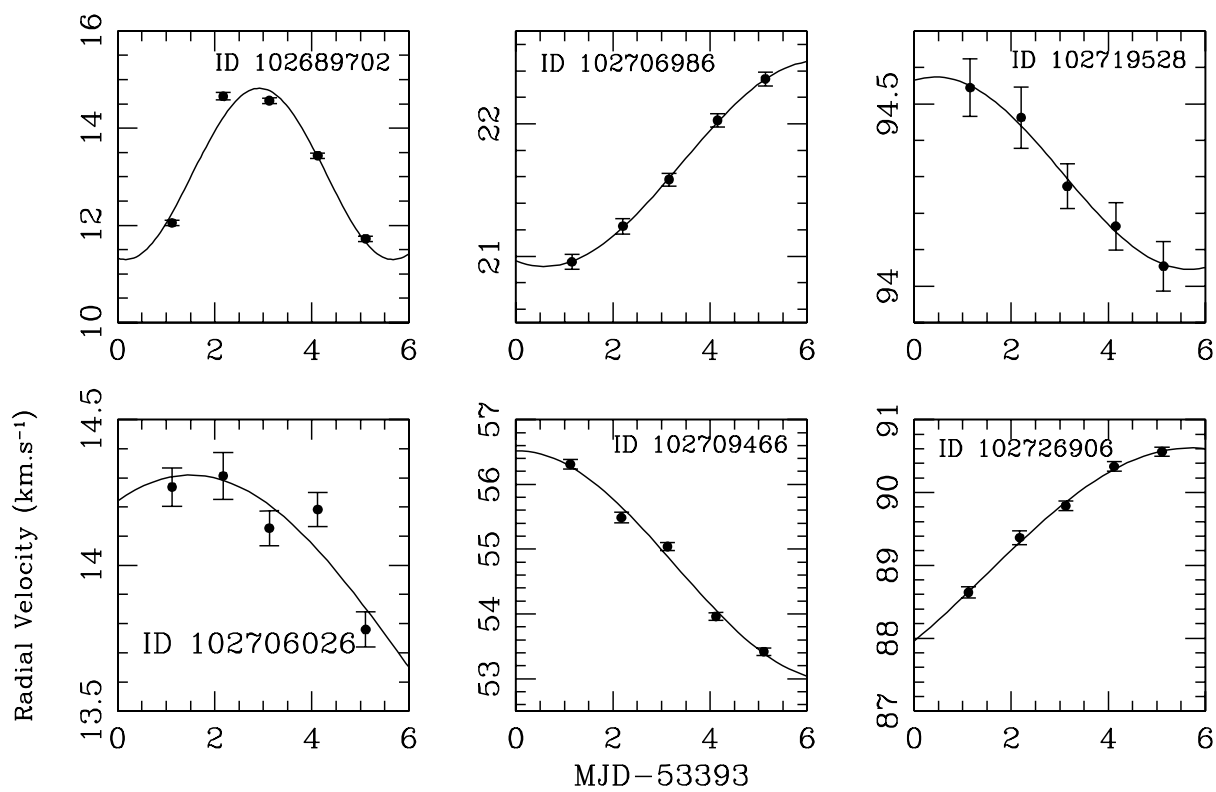

(b)
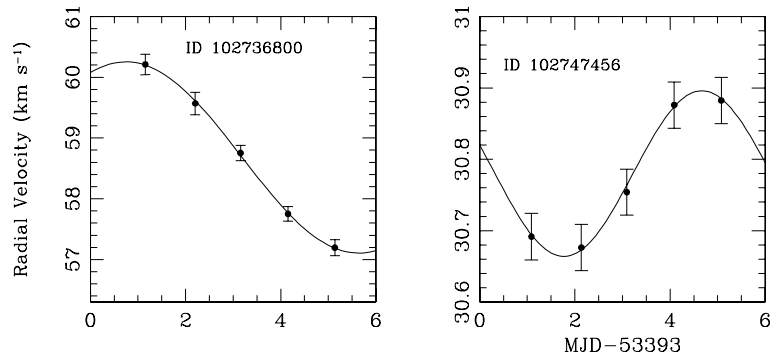

(c)

Fig. 5. Sinusoidal fits of the 14 exoplanet and brown dwarf candidates. The RV measurements are represented with their error bars and the curve represents the best sinusoidal fit.

corresponds to the value on the $x$-axis of the $\sigma$ curve for the defined detection threshold on the $y$-axis. We then computed the dispersion and estimated the detection probabilities of such systems as a function of the detection threshold. Only circu- lar orbital cases were considered and we did not include a distribution of orbital inclinations. In these simulations, we only considered the induced RV motion of a star that a planet im- 
Table 5. Brown dwarf and exoplanet candidate hosting stars. The spectral types and luminosity classes are determined from our spectral analysis carried out with MATISSE and with the photometric analysis.

\begin{tabular}{cccccc}
\hline \hline $\begin{array}{c}\text { CoRoT } \\
\text { ID }\end{array}$ & $\begin{array}{c}\alpha \\
(\mathrm{h} \mathrm{mn} \mathrm{s}) \\
(\mathrm{J} 200)\end{array}$ & $\begin{array}{c}\delta \\
(\mathrm{d} \mathrm{mn} \mathrm{s}) \\
(\mathrm{J} 2000)\end{array}$ & $m_{V}$ & $\begin{array}{c}\text { Spectral type } \\
\text { of the parent star } \\
\text { (photometric analysis })\end{array}$ & $\begin{array}{c}\text { Spectral type } \\
\text { of the parent star } \\
\text { (MATISSE analysis })\end{array}$ \\
\hline 102636650 & 64218.87 & -12406.12 & 15.16 & K0V & G8V \\
102638570 & 64221.35 & -11147.58 & 12.27 & K4III & K3III \\
102638956 & 64221.88 & -03150.74 & 13.52 & G2V & G2IV \\
102660283 & 64249.20 & 01759.78 & 12.17 & K3III & G8III \\
102664207 & 64254.18 & -12037.79 & 15.15 & F6V & F8V \\
102666192 & 64256.67 & 01922.08 & 14.62 & G2V & F5V \\
102689702 & 64325.98 & 04832.9 & 13.90 & F5V & F8V \\
102706026 & 64345.86 & -05031.60 & 14.45 & G2V & F8V \\
102706986 & 64347.08 & 02350.14 & 14.04 & G2V & F8V \\
102709466 & 64350.51 & 05003.52 & 14.80 & G2V & G1V \\
102719528 & 64404.62 & 03631.57 & 15.19 & G0III & G0IV \\
102726906 & 64415.39 & 05343.22 & 15.39 & G0III & G7IV \\
102736800 & 64428.79 & 03521.73 & 15.25 & G0III & No output \\
102747456 & 64443.05 & -12213.84 & 11.90 & K4III & K3III \\
\hline
\end{tabular}

Table 6. Estimated orbital parameters of the brown dwarf and exoplanet candidates, average photon-noise uncertainty and residuals of the sinusoidal and linear fits. We quadratically added systematic errors of $30 \mathrm{~m} \mathrm{~s}^{-1}$ to obtain the global error on the measurements. The estimated mass of the parent star based on the MATISSE analysis and the companion candidate are also given. The targets in bold type are our best candidates obtained by comparing the residuals from both solutions.

\begin{tabular}{|c|c|c|c|c|c|c|c|c|}
\hline $\begin{array}{c}\text { CoRoT } \\
\text { ID }\end{array}$ & $\begin{array}{c}K \\
\left(\mathrm{~km} \mathrm{~s}^{-1}\right)\end{array}$ & $\begin{array}{c}P \\
\text { (days) }\end{array}$ & $\begin{array}{c}V_{0} \\
\left(\mathrm{~km} \mathrm{~s}^{-1}\right)\end{array}$ & $\begin{array}{c}\text { Average } \\
\text { photon-noise uncertainty } \\
\left(\mathrm{m} \mathrm{s}^{-1}\right)\end{array}$ & $\begin{array}{c}\text { Residuals from } \\
\text { a sinusoidal fit } \\
\left(\mathrm{m} \mathrm{s}^{-1}\right)\end{array}$ & $\begin{array}{l}\text { Residuals from } \\
\text { a linear fit } \\
\left(\mathrm{m} \mathrm{s}^{-1}\right)\end{array}$ & $\begin{array}{l}\text { Estimated mass } \\
\text { of the parent star } \\
\qquad\left(M_{\odot}\right)\end{array}$ & $\begin{array}{l}\text { Estimated } \\
M_{2} \sin (i) \\
\left(M_{\text {Jup }}\right)\end{array}$ \\
\hline 102636650 & 0.32 & 8.1 & 10.42 & 44.3 & 47.1 & 57.7 & 0.9 & 3.0 \\
\hline 102638570 & 0.13 & 6.5 & 66.14 & 9.3 & 6.6 & 39.3 & 2.2 & 2.1 \\
\hline 102638956 & 0.09 & 9.4 & 48.9 & 14.1 & 19.5 & 21.0 & 1.0 & 1.0 \\
\hline 102660283 & 1.13 & 11.2 & 67.56 & 14.8 & 16.7 & 55.8 & 2.4 & 23.4 \\
\hline 102664207 & 0.72 & 11.2 & 33.97 & 55.5 & 0.7 & 75.8 & 1.2 & 9.4 \\
\hline 102666192 & 0.34 & 4.5 & 30.19 & 52.8 & 0.8 & 156.3 & 1.3 & 3.4 \\
\hline 102689702 & 1.76 & 5.5 & 13.01 & 34.2 & 231.8 & 1200 & 1.2 & 18.1 \\
\hline 102706026 & 0.6 & 18.0 & 13.7 & 38.2 & 80.0 & 108.2 & 1.2 & 8.7 \\
\hline 102706986 & 0.78 & 11.2 & 21.70 & 28.9 & 8.9 & 44.0 & 1.2 & 10.2 \\
\hline 102709466 & 1.77 & 13.1 & 54.76 & 39.1 & 110.4 & 113.9 & 1.1 & 22.9 \\
\hline 102719528 & 0.26 & 10.5 & 94.31 & 43.1 & 15.4 & 22.8 & 1.6 & 4.0 \\
\hline 102726906 & 1.74 & 16.9 & 89.04 & 44.5 & 40.8 & 120.3 & 1.5 & 30.2 \\
\hline 102736800 & 1.57 & 9.8 & 58.68 & 95.4 & 27.5 & 101.2 & 2.1 & 28.4 \\
\hline 102747456 & 0.12 & 5.8 & 30.78 & 8.0 & 4.6 & 32.6 & 2.2 & 1.8 \\
\hline
\end{tabular}

plies and compared it to the detection threshold obtained for the GIRAFFE instrument.

\subsubsection{For binary systems}

We considered a detection threshold of $0.2 \mathrm{~km} \mathrm{~s}^{-1}$ and calculated the detection probabilities for 3 different stellar companion masses $\left(M_{2} / M_{1}=0.5,0.3\right.$ and $\left.0.1 M_{\odot}\right)$. Using the $\sigma$ curve we determined that such a detection threshold corresponds to a photon-noise uncertainty of $90 \mathrm{~m} \mathrm{~s}^{-1}$ which covers about $75 \%$ of our stellar sample when considered as an upper limit. The simulation results are presented in Fig. 6. These simulations clearly show that we can identify binary stars with periods longer than 100 days in our observational configuration.

Among the 701 stars with detected CCF peaks we identified 50 binary stars. Assuming that $50 \%$ of the stars are binaries and that $13 \%$ of this population have an orbital period less than 100 days (Duquennoy \& Mayor 1991), we expected to detect 46 of these systems. This is in good agreement with our results. We demonstrated above that the detection of systems with an orbital period of up to 1000 days is possible. Following the statistics from Duquennoy \& Mayor (1991), we would expect to detect a sample of 90 binary stars with an orbital period less than 1000 days. This suggests that some of the identified binary stars may have an orbital period longer than 100 days. The detection ability indicates indeed that we cover a small part of the systems with an orbital period from 100 to 1000 days. As suggested in Sect. 5.2.2 some of the radial velocity variations of our candidates suggest longer period binary systems. The number of RV measurements indeed limits the efficiency of the detection and a precise determination of the period.

\subsubsection{For low-mass companions}

For sub-stellar companions, we calculated the detection probabilities for 3 different companion masses $\left(M_{2}=1,3\right.$ and $\left.5 M_{\text {Jup }}\right)$ and a detection threshold of $100 \mathrm{~m} \mathrm{~s}^{-1}$. Using the $\sigma$ curve we determined that such a detection threshold corresponds to a photon-noise uncertainty of $37 \mathrm{~m} \mathrm{~s}^{-1}$ on the $x$-axis. Notice that only $25 \%$ of our sample of stars actually match this lower limit value. We computed the detection probabilities for objects with an orbital period ranging from 1.2 to 20 days. Our simu- 


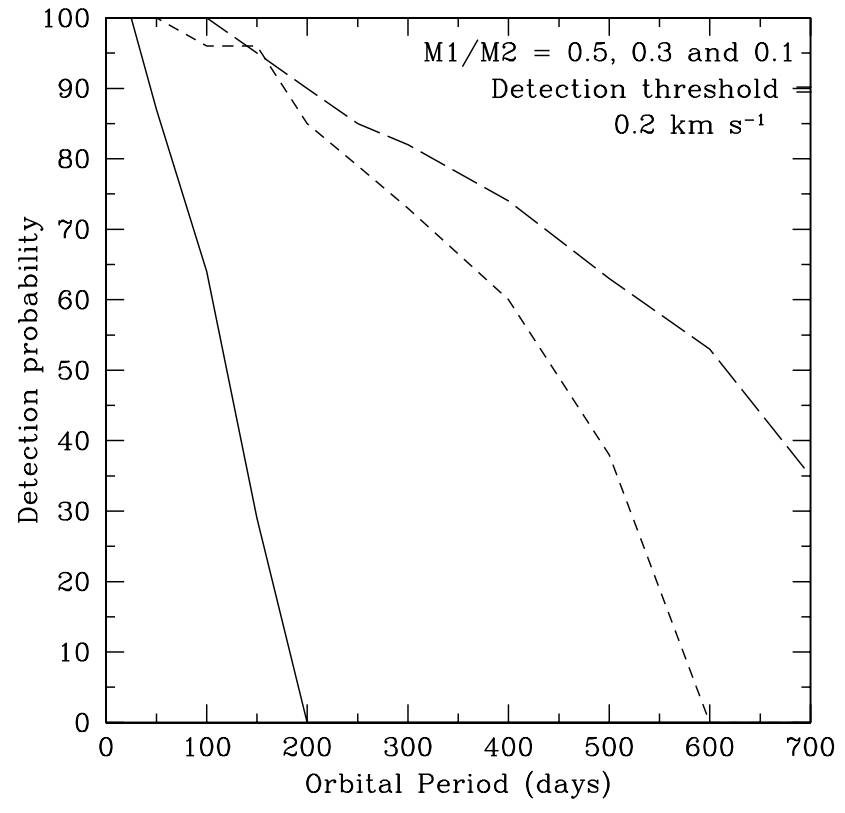

Fig. 6. Detection probability of massive stellar companions as a function of the orbital period for stellar companion masses of $0.1,0.3$ and $0.5 M_{\odot}$ and a solar-mass parent star and for a detection threshold of $0.2 \mathrm{~km} \mathrm{~s}^{-1}$. The solid line represents the $M_{1} / M_{2}=0.1 M_{\odot}$ case, the short-dashed line stands for the $M_{1} / M_{2}=0.3 M_{\odot}$ case and the longdashed line stands for the $M_{1} / M_{2}=0.5 M_{\odot}$ case.

lations show in Fig. 7 that we have a $100 \%$ probability of detecting companions of 1,3 and $5 M_{\text {Jup }}$ with a period less than 2, 9 and 12 days respectively. The simulations also show that the probabilities of detecting companions with less than a Jupiter mass are weak and rapidly decrease with the orbital period, with such a detection threshold and a small number of RV measurements. However this suggests that close-in massive and supermassive giant planets ( $>3 M_{\text {Jup }}$ ) could be detected.

As detailed in Butler et al. (2006), 1.2\% of solar-type stars host at least one hot-Jupiter with a period less than 10 days. Considering our sample of 584 targets with a distinct peak in their CCF and 5 measurements, we should expect to detect about 7 hot Jupiters. The statistics of known exoplanets suggest that $33 \%$ of them have a mass greater than $1 M_{\text {Jup }}$. With only $25 \%$ of our sample (146 stars), with our observational strategy, our survey is expected to detect one massive exoplanet.

\subsection{Extended ability for exoplanet search with the GIRAFFE spectrograph}

We have shown that the GIRAFFE multi-fiber spectrograph could be used to carry out large exoplanet surveys. Indeed, with a few RV measurements a systematic error floor of $30 \mathrm{~m} \mathrm{~s}^{-1}$ could be achieved, allowing one to explore the massive and the hotJupiter exoplanet populations. With our observational strategy, only $25 \%$ of the observed stars have a photon-noise uncertainty less than the one obtained for a detection threshold of $100 \mathrm{~m} \mathrm{~s}^{-1}$. However this limitation might be overcome. For example, increasing the number of RV consecutive measurements from 5 to 10 could reduce the detection threshold to $1.7 \sigma$. At small photon-noise uncertainties the detection threshold is equal to $50 \mathrm{~m} \mathrm{~s}^{-1}$. In that case it would be possible to detect a Jupitermass companion with an orbital period up to 12 days. Another solution would be to multiply the exposure time of such faint stars by a factor of two. As a result, the photon-noise uncertainty

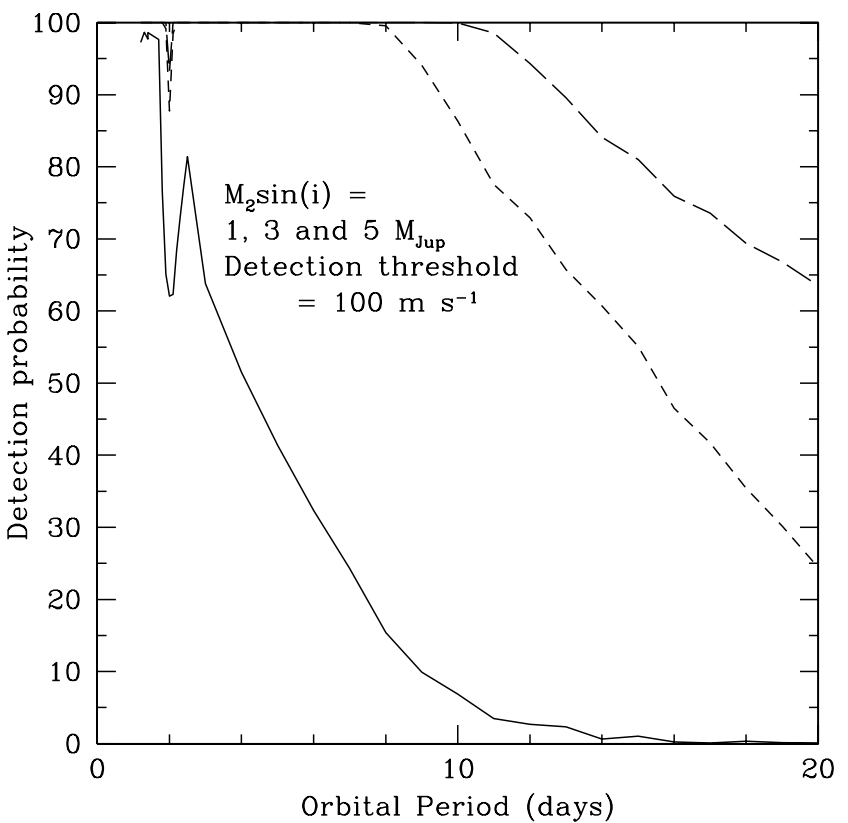

Fig. 7. Detection probability of exoplanets as a function of the orbital period for 4 different companion masses $\left(M_{1} / M_{2}=1,3\right.$ and $\left.5 M_{\mathrm{Jup}}\right)$, a solar-mass parent star and a fixed detection threshold of $100 \mathrm{~m} \mathrm{~s}^{-1}$. The solid, short-dashed and long-dashed lines represent the different probabilities for companion masses of 1,3 and $5 M_{\text {Jup. Note that the }}$ drop in the detection probability of a $1 M_{\text {Jup }}$ companion around 2 days decreases due to a stroboscopic effect of our exact simulated sampling of one day.

would be divided by $\sqrt{2}$, allowing one to widen the detection threshold of $100 \mathrm{~m} \mathrm{~s}^{-1}$ to $50 \%$ of the sample. In a similar way, observing a sample of stars brighter by about one magnitude would lead to a similar result. Finally, a multi-fiber facility with systematic errors of $20 \mathrm{~m} \mathrm{~s}^{-1}$ would allow us to decrease the detection threshold to $35 \mathrm{~m} \mathrm{~s}^{-1}$ at small photon-noise uncertainties, giving access to the $0.5 M_{\text {Jup }}$ exoplanets with short periods. With $10 \mathrm{RV}$ measurements, objects with periods of up to 8 days would be detected. Such an efficiency would allow for the discovey of up to 5 new hot-Jupiters in only 10 half-nights.

\section{Conclusion}

A multi-object RV survey enables a very good investigation of a large sample of stars by distinguishing binaries and low-mass companion candidates. With only 5 consecutive RV measurements, with the GIRAFFE instrument we achieved a systematic $\mathrm{RV}$ error of $30 \mathrm{~m} \mathrm{~s}^{-1}$ on 584 stars. This RV precision reached is 5 times less than announced by the ESO. On a sample of 816 stars we identified 50 binary systems, 14 exoplanet and brown dwarf candidates, 9 active stars or blended binary stars and 5 unsolved cases. Given the existence of the BD desert, most of our candidates might be long-period binary stars. Assuming the statistics on exoplanets (Butler et al. 2006; Schneider 2007, url: exoplanet .eu) we could expect one confirmed exoplanet. However, with only 5 RV measurements, we could not derive the exact orbital parameters of our candidates and further higher precision measurements are needed. With this strategy we reduced by a factor of about 50 a large sample of stars to a much smaller sample with interesting RV variations for the search of massive hot Jupiter in only 5 consecutive half-nights. This study demonstrates that this approach (with optimized data reduction) is very efficient in searching for massive exoplanets and brown 
dwarfs and could bring a key contribution to large and very large surveys. A similar program carried out with a mono-fiber instrument would require at least one complete year of observations.

The space mission CoRoT was launched successfully on December 2006. This multi-fiber approach could be very useful and efficient in the follow-up of the large sample of stars that will be observed by the CoRoT satellite. Indeed a precision of $30 \mathrm{~m} \mathrm{~s}^{-1}$ obtained with only a few measurements will allow us to carry out radial velocity observations over a large sample of stars. Such observations are necessary to further discriminate binaries, low-mass candidates and the hot-Jupiter population. In order to characterize the hot-Jupiter population, the previous knowledge of the two orbital parameters $\mathrm{P}$ and $\mathrm{T}_{0}$ derived from previous high precision photometric observations will significantly reduce the detection threshold and allow us to determine the parameters $K$ and $V_{0}$ at the $1 \sigma$ level. The selection made with such a multi-fiber instrument leads to a significant reduction of the on-telescope time. The most interesting candidates could thus be observed with more accurate instruments in a second step.

New measurements are now needed to confirm the nature of our substellar companions and derive accurate orbital parameters. To that purpose we will use the new spectrograph SOPHIE (Bouchy \& The Sophie Team 2006), mounted on the 193-cm telescope at the Observatoire de Haute Provence.

Acknowledgements. N.C.S. would like to thank the Fundao para a Cincia e a Tecnologia, Portugal, for the grant (reference POCI/CTE-AST/56453/2004). This work was supported in part by the EC's FP6 and by FCT (with POCI2010 and FEDER funds), within the HELAS international collaboration.

\section{References}

Alonso, R., Brown, T. M., Charbonneau, D., et al. 2007, in Transiting Extrasolar Planets Worshop, ed. C. Alfonso, D. Weldrake, \& Th. Henning, ASP Conf. Ser., 366, 13

Baglin, A. 2003, Adv. Space Res., 31, 345

Bakos, G., Noyes, R. W., Latham, D. W., et al. 2006, in Tenth Anniversary of 51 Peg-b: Status of and prospects for hot Jupiter studies, ed. L. Arnold, F. Bouchy, \& C. Moutou, 184

Bakos, G. A., Kovacs, G., Torres, G., et al. 2007, ArXiv e-prints, 705

Baranne, A., Queloz, D., Mayor, M., et al. 1996, A\&AS, 119, 373

Blecha, A., Cayatte, V., North, P., Royer, F., \& Simond, G. 2000, in Optical and IR Telescope Instrumentation and Detectors, ed. M. Iye, \& A. F. Moorwood, Proc. Spie 4008, 467
Bouchy, F., \& The Sophie Team. 2006, in Tenth Anniversary of 51 Peg-b: Status of and prospects for hot Jupiter studies, ed. L. Arnold, F. Bouchy, \& C. Moutou, 319

Bouchy, F., Pont, F., Santos, N. C., et al. 2004, A\&A, 421, L13

Bouchy, F., Pont, F., Melo, C., et al. 2005, A\&A, 431, 1105

Butler, R. P., Marcy, G. W., Williams, E., Hauser, H., \& Shirts, P. 1997, ApJ, 474, L115

Butler, R. P., Wright, J. T., Marcy, G. W., et al. 2006, ApJ, 646, 505

Chabrier, G., Baraffe, I., Allard, F., \& Hauschildt, P. 2000, ApJ, 542, 464

Cox, A. N. 2000, Allen's astrophysical quantities, 4th Ed. (New York: AIP Press; Springer), ed. A. N. Cox.

Deleuil, M. 2006, ESA SP-1306, in The CoRoT mission pre-launch status, Stellar Seismology and Planet Finding, ed. A. Baglin, J. Lochard, M. Fridlund, \& L. Conroy

Duquennoy, A., \& Mayor, M. 1991, A\&A, 248, 485

Fischer, D. A., Marcy, G. W., Butler, R. P., Vogt, S. S., \& Apps, K. 1999, PASP, 111,50

Goldreich, P., \& Tremaine, S. 1980, ApJ, 241, 425

Gray, D. F. 1988, Lectures on spectral-line analysis: F, G, and K stars (Arva: Ontario Gray)

Grether, D., \& Lineweaver, C. H. 2006, ApJ, 640, 1051

Halbwachs, J. L., Mayor, M., \& Udry, S. 2005, A\&A, 431, 1129

Horne, K. 1986, PASP, 98, 609

Lin, D. N. C., Bodenheimer, P., \& Richardson, D. C. 1996, Nature, 380, 606

Marcy, G., Butler, R. P., Fischer, D., et al. 2005, Progr. Theor. Phys. Suppl., 158, 24

Pasquini, L., Avila, G., Allaert, E., et al. 2000, in Optical and IR Telescope Instrumentation and Detectors, ed. M. Iye, \& A. F. Moorwood, Proc. SPIE 4008, 129

Pepe, F., Mayor, M., Galland, F., et al. 2002, A\&A, 388, 632

Pollacco, D. L., Skillen, I., Cameron, A. C., et al. 2006, PASP, 118, 1407

Pont, F., Bouchy, F., Melo, C., et al. 2005, A\&A, 438, 1123

Pont, F., Moutou, C., Bouchy, F., et al. 2006, A\&A, 447, 1035

Queloz, D., Mayor, M., Weber, L., et al. 2000, A\&A, 354, 99

Queloz, D., Henry, G. W., Sivan, J. P., et al. 2001, A\&A, 379, 279

Rauer, H., Eislöffel, J., Erikson, A., et al. 2004, PASP, 116, 38

Recio-Blanco, A., Bijaoui, A., \& de Laverny, P. 2006, MNRAS, 370, 141

Royer, F., Blecha, A., North, P., et al. 2002, in Astronomical Data Analysis II, ed. J.-L. Starck, F. Murtagh, D. Proc. Spie, 4847, 184, ed. J.-L. Starck \& F. D. Murtagh, 184

Santos, N. C., Mayor, M., Naef, D., et al. 2002, A\&A, 392, 215

Udalski, A., Paczynski, B., Zebrun, K., et al. 2002, Acta Astron., 52, 1

Udry, S., Mayor, M., Naef, D., et al. 2002, A\&A, 390, 267

Wilkinson, M. I., Vallenari, A., Turon, C., et al. 2005, MNRAS, 359, 1306

Wright, J. T., Marcy, G. W., Fischer, D. A., et al. 2006, ArXiv Astrophysics e-prints 